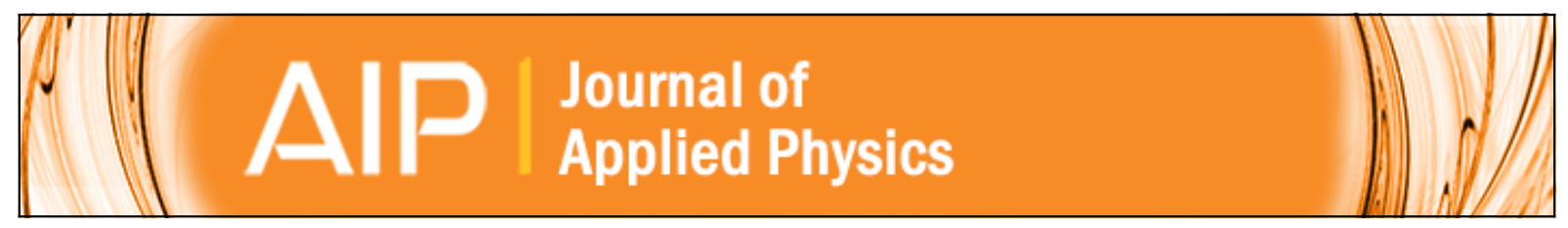

\title{
Room-temperature ferromagnetism in manganese doped reduced rutile titanium dioxide thin films
}

Zhenjun Wang, Jinke Tang, Yuxi Chen, Leonard Spinu, Weilie Zhou, and Le Duc Tung

Citation: Journal of Applied Physics 95, 7384 (2004); doi: 10.1063/1.1667858

View online: http://dx.doi.org/10.1063/1.1667858

View Table of Contents: http://scitation.aip.org/content/aip/journal/jap/95/11?ver=pdfcov

Published by the AIP Publishing

\section{Articles you may be interested in}

Defect-mediated room temperature ferromagnetism in vanadium dioxide thin films

Appl. Phys. Lett. 95, 102506 (2009); 10.1063/1.3224202

Room temperature ferromagnetism in transition metal $(\mathrm{V}, \mathrm{Cr}, \mathrm{Ti})$ doped In 203

J. Appl. Phys. 101, 09 N513 (2007); 10.1063/1.2712018

Ferromagnetism in chromium-doped reduced-rutile titanium dioxide thin films

J. Appl. Phys. 95, 7381 (2004); 10.1063/1.1667806

Ferromagnetism and transport properties of Fe-doped reduced-rutile TiO $2-\delta$ thin films

J. Appl. Phys. 93, 7870 (2003); 10.1063/1.1556122

Semiconducting and ferromagnetic behavior of sputtered Co-doped TiO 2 thin films above room temperature J. Appl. Phys. 91, 8093 (2002); 10.1063/1.1452650
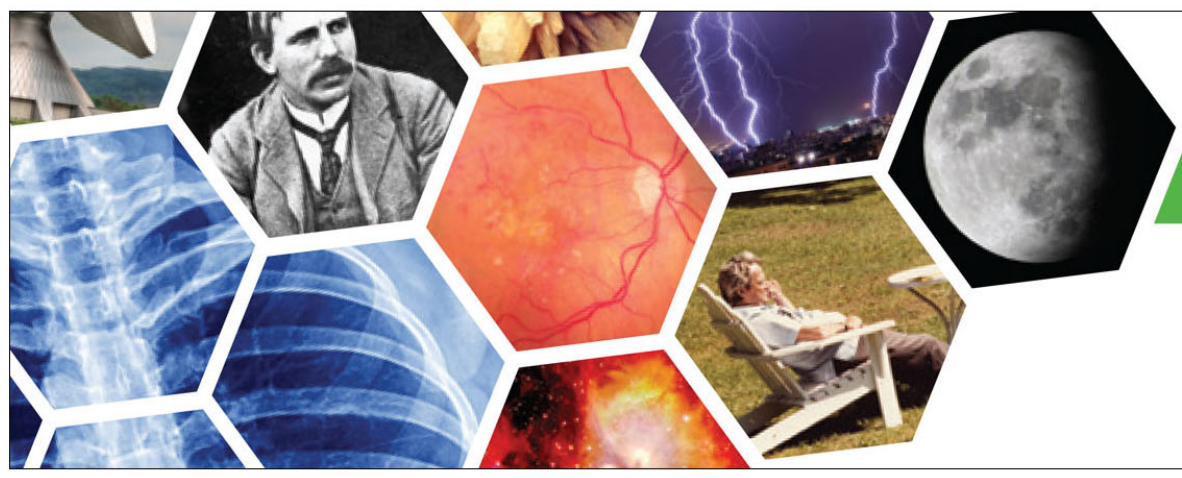

\section{SUBSCRIBE TO




\title{
Room-temperature ferromagnetism in manganese doped reduced rutile titanium dioxide thin films
}

\author{
Zhenjun Wang and Jinke Tang ${ }^{\text {a) }}$ \\ Department of Physics, University of New Orleans, New Orleans, Louisiana 70148 \\ Yuxi Chen, Leonard Spinu, and Weilie Zhou \\ Advanced Materials Research Institute, University of New Orleans, New Orleans, Louisiana 70148 \\ Le Duc Tung \\ Department of Physics, University of Warwick, Coventry CV4 7AL, United Kingdom
}

(Presented on 9 January 2004)

\begin{abstract}
$\mathrm{Mn}_{x} \mathrm{Ti}_{1-x} \mathrm{O}_{2-\delta}(x=0.02-0.12)$ thin films grown on $\alpha-\mathrm{Al}_{2} \mathrm{O}_{3}$ substrates by pulsed-laser deposition have been investigated. X-ray diffraction and transmission electron microscopy results indicate that the films are single phase and reduced rutile-type. Superconducting quantum interference device magnetometer measurements show the films are ferromagnetic at room temperature with nonzero coercivity up to $170 \mathrm{Oe}$. The saturation magnetization of the reduced films is as high as $0.83 \mu_{\mathrm{B}}$ per $\mathrm{Mn}$ atom at room temperature. The temperature dependence of the resistivity shows semiconducting behavior with $p$-type carriers. The nature of the $p$-type conduction and its significance to the ferromagnetism are discussed. (c) 2004 American Institute of Physics. [DOI: 10.1063/1.1667858]
\end{abstract}

\section{INTRODUCTION}

The ferromagnetism in semiconductors $(\mathrm{Ga}, \mathrm{Mn}) \mathrm{As}$ and (In,Mn)As has been well established. The Curie temperatures are $\sim 110 \mathrm{~K}$ for $(\mathrm{Ga}, \mathrm{Mn})$ As (Ref. 1) and $\sim 35 \mathrm{~K}$ for (In,Mn)As. ${ }^{2}$ Recent results suggest that $(\mathrm{Ga}, \mathrm{Mn}) \mathrm{N},{ }^{3-6}$ (Ga,Mn)P:C, ${ }^{7,8} \mathrm{CdMnGeP}_{2},{ }^{9} \quad\left(\mathrm{Zn}_{1-x} \mathrm{Mn}_{x}\right) \mathrm{GeP}_{2},{ }^{10,11}$ and Co-doped $\mathrm{ZnO}$ (Ref. 12) are ferromagnetic at room temperature. It has been reported that a ferromagnetic state exists in Co-doped anatase/rutile $\mathrm{TiO}_{2}$ at room temperature. ${ }^{13-17}$ Very recently, we have reported room temperature ferromagnetism in $\mathrm{Fe}$ doped reduced-rutile $\mathrm{TiO}_{2} \cdot{ }^{18}$ Strong evidences were given to show that the observed ferromagnetism is intrinsic to the doped films. The carriers of the Fe-doped reducedrutile are $p$-type, which is different from the $n$-type found in Co-doped $\mathrm{TiO}_{2} \cdot{ }^{13}$

In this study, we report the magnetic properties of $\mathrm{Mn}_{x} \mathrm{Ti}_{1-x} \mathrm{O}_{2-\delta}(x=0.02-0.12)$ thin films of reduced-rutile structure. ${ }^{18-21} \mathrm{Mn}$ doping in reduced rutile leads to a ferromagnetic state at room temperature.

\section{EXPERIMENT}

$\mathrm{Mn}_{x} \mathrm{Ti}_{1-x} \mathrm{O}_{2}(x=0.02-0.12)$ thin films were grown on $\alpha-\mathrm{Al}_{2} \mathrm{O}_{3} \quad$ (012) substrates by pulsed-laser deposition. $\mathrm{Mn}_{x} \mathrm{Ti}_{1-x} \mathrm{O}_{2}$ targets were prepared using standard ceramic synthesis techniques. The films were prepared in vacuum at a substrate temperature of $970 \mathrm{~K}$. The deposition rate was between 0.1 and $0.3 \AA / s$, and the film thickness varied from 40 to $100 \mathrm{~nm}$.

The crystal structure of the films was investigated by $\mathrm{x}$-ray diffraction (XRD) with $\mathrm{Cu}-\mathrm{K} \alpha$ radiation and transmission electron microscopy (TEM). The magnetic properties of the samples were determined from a superconducting quantum interference device magnetometer. The transport proper-

a)Electronic mail: jtang@uno.edu ties were measured with a physical property measurement system from Quantum Design (San Diego, CA).

\section{RESULTS AND DISCUSSION}

The XRD results show that the films are single-phase reduced-rutile. A typical XRD pattern of $\mathrm{Mn}_{x} \mathrm{Ti}_{1-x} \mathrm{O}_{2}$ thin films is shown in Fig. 1(a). The film with $x=0.08$, $\mathrm{Mn}_{0.08} \mathrm{Ti}_{0.92} \mathrm{O}_{2-\delta}$, is of reduced-rutile type with (202) plane, referred to the stoichiometric rutile tetragonal cell, parallel to the film plane as shown in Fig. 1(a). The two vertical lines show (101) and (202) reflections of the stoichiometric rutile. The (202) peak in Fig. 1 is shifted toward lower $2 \theta$ angles by about $0.65^{\circ}$. The (101) peak is absent in sample $x=0.08$ due to substantial reduction that forms Magneli shear plane..$^{20,21}$ The XRD results of the reduced-rutile $\mathrm{Mn}_{x} \mathrm{Ti}_{1-x} \mathrm{O}_{2-\delta}$ films are similar to that of $\mathrm{Fe}$ doped reduced-rutile $\mathrm{TiO}_{2} .{ }^{18} \mathrm{TEM}$ observation indicates no sign of segregation of impurity phase in the samples, as shown in Fig. 1(b).

The magnetization versus applied field (M-H) curves of all the films measured at $300 \mathrm{~K}$ show hysteresis with nonzero coercivity. Figure 2 shows the magnetic hysteresis loops for doped films $x=0.06,0.08$, and 0.12 . The inset shows the low field region of the hysteresis loop for $x=0.08$. The hysteresis loops indicate that the Mn-doped reduced-rutile films are ferromagnetic at $300 \mathrm{~K}$. The magnetic moment per $\mathrm{Mn}$ increases initially with the $\mathrm{Mn}$ concentration. It peaks at $x$ $=0.08$ and decreases upon further increase of the Mn doping level. The room temperature saturation moment is $0.65,0.83$, and $0.14 \mu_{\mathrm{B}}$ per $\mathrm{Mn}$ atom for $x=0.06,0.08$, and 0.12 , respectively. The coercivity of the three films follows a similar trend, $H_{c}=85,170$, and 110 Oe at room temperature.

It is not difficult to rule out any impurity cause for the magnetic signals in the Mn-doped samples because Mn and Mn oxides, including Ti/Mn oxides, are not ferromagnetic except $\mathrm{Mn}_{3} \mathrm{O}_{4}$ and perhaps certain $\mathrm{Mn}-\mathrm{Ti}^{22}$ But the latter two have Curie temperatures in the $40 \mathrm{~K}$ range, too low to 

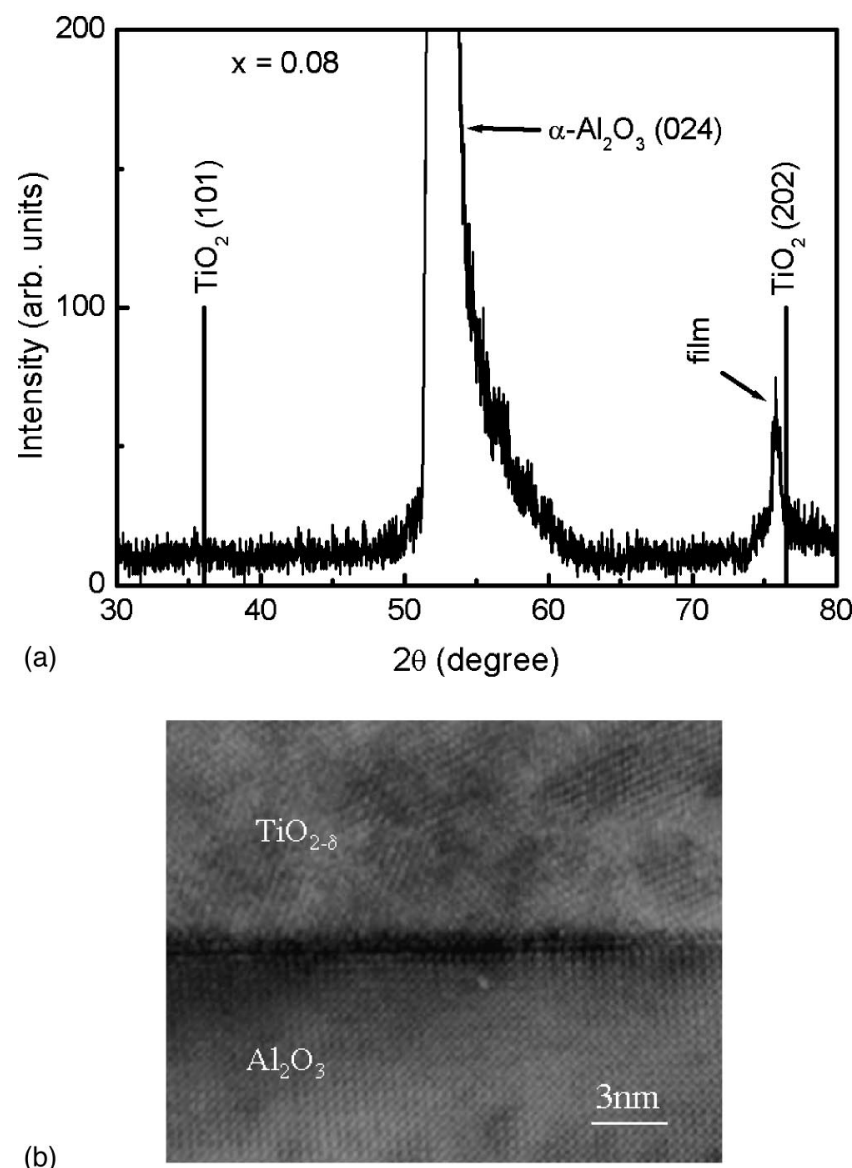

(b)

FIG. 1. (a) XRD pattern of a $\mathrm{Mn}_{0.08} \mathrm{Ti}_{0.92} \mathrm{O}_{2-\delta}$ film grown on $\alpha-\mathrm{Al}_{2} \mathrm{O}_{3}(012)$ substrate showing reduced rutile structure and (b) TEM image for the same film.

account for the room temperature ferromagnetism. Mn, $\mathrm{MnO}, \mathrm{Mn}_{2} \mathrm{O}_{3}, \mathrm{MnO}_{2}$, and $\mathrm{MnTiO}_{3}$ are antiferromagnetic with Néel temperatures of $\sim 100 \mathrm{~K}$ or less. The low Néel temperatures rule out the possibility that parasitic magnetism in these antiferromagnets contributes to the room temperature ferromagnetic signals. Furthermore, the magnetic moment of parasitic magnets would be far less than observed in our samples (as high as $0.83 \mu_{\mathrm{B}} / \mathrm{Mn}$ ).

The temperature dependence of the magnetization for sample $x=0.08$ measured with a field of 300 Oe is shown in Fig. 3. The magnetization is essentially temperature independent over the temperature range shown except at very low temperatures. The Curie temperature $T_{\mathrm{c}}$ of the films is above $300 \mathrm{~K}$. Although the room temperature ferromagnetism in the Mn-doped reduced-rutile is not understood, it is expected to share the same origin as that of Fe-doped reduced-rutile. It may be related to the Zener-type hole-mediated RudermanKittel-Kasuya-Yosida- (RKKY) -like interaction, which is responsible for the well-established $I I I-V$ magnetic semiconductors $(\mathrm{Ga}, \mathrm{Mn}) \mathrm{As}^{23-25}$ So far experimental values of the magnetization are only a fraction (from $1 / 7$ to $1 / 2$ ) of the expected value of $5 \mu_{\mathrm{B}} / \mathrm{Mn}$ in doped GaAs. ${ }^{28,29}$ There are several possible reasons for the magnetization deficit, but it is most likely that the local electronic structure associated with certain defects precludes individual $\mathrm{Mn}$ moments from participating in the ferromagnetism. ${ }^{26,27} \mathrm{We}$ expect a similar

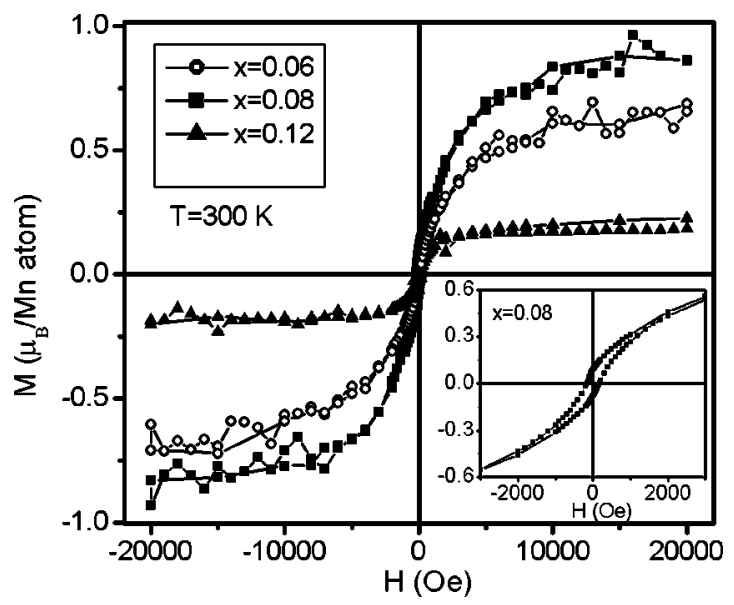

FIG. 2. Magnetization vs field curves of $\mathrm{Mn}_{x} \mathrm{Ti}_{1-x} \mathrm{O}_{2-\delta}$ films measured with a SQUID at room temperature. Inset shows the low field region of the hysteresis loop for $x=0.08$.

situation in our samples. The upturn at low temperature shown in Fig. 3 is most likely attributed to the Mn moments that remain paramagnetic. In Fig. 2, the decrease of the magnetic moment at high Mn concentration $(x=0.12)$ is possibly due to the increased antiferromagnetic superexchange interaction between $\mathrm{Mn}$ moments which, in general, leads to a spin-glass-like state as observed in many $I I-V I$ dilute magnetic semiconductors above $20 \% \mathrm{Mn}^{28,29}$

All reduced-rutile films exhibit semiconducting behavior. Figure 4 shows the temperature dependence of the resistivity for films $x=0.06,0.08$, and 0.12 . It increases with decreasing temperature and with increasing Mn concentration. The resistivity at low temperature is higher than that of Fe-doped reduced-rutile $\mathrm{Fe}_{x} \mathrm{Ti}_{1-x} \mathrm{O}_{2-\delta} \cdot{ }^{18}$

Hall measurements indicate that the carriers are $p$-type with a carrier density of $6 \times 10^{21} / \mathrm{cm}^{3}$ at room temperature for sample $x=0.08$. The theory of Dietl et al. suggests that holes are essential for the RKKY induced ferromagnetic state to stabilize at room temperature. ${ }^{23}$ The large hole concentration in our samples may have played an important role in the coupling between the Mn magnetic moments that gives rise to the room temperature ferromagnetism.

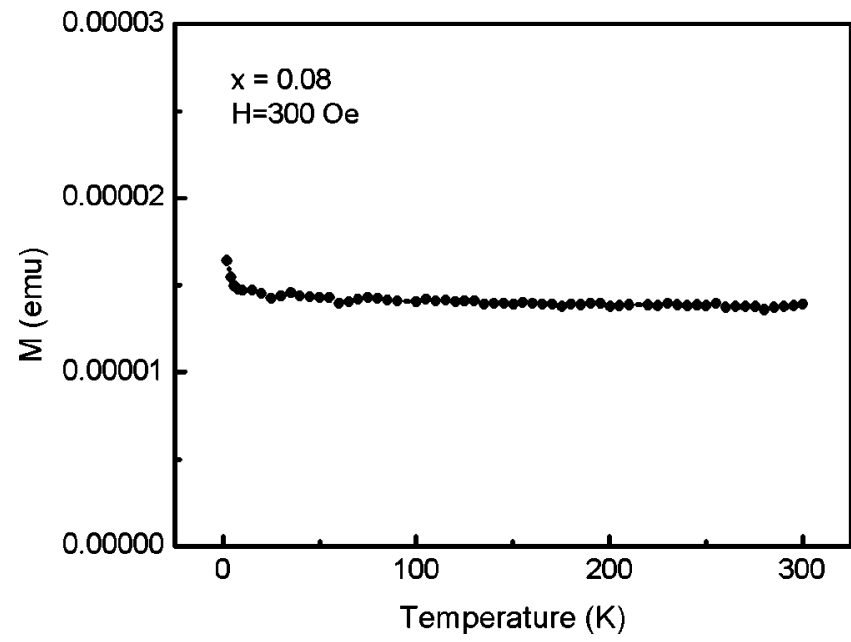

FIG. 3. Magnetization as a function of temperature in a field of 300 Oe. The Curie temperature is above $300 \mathrm{~K}$. 


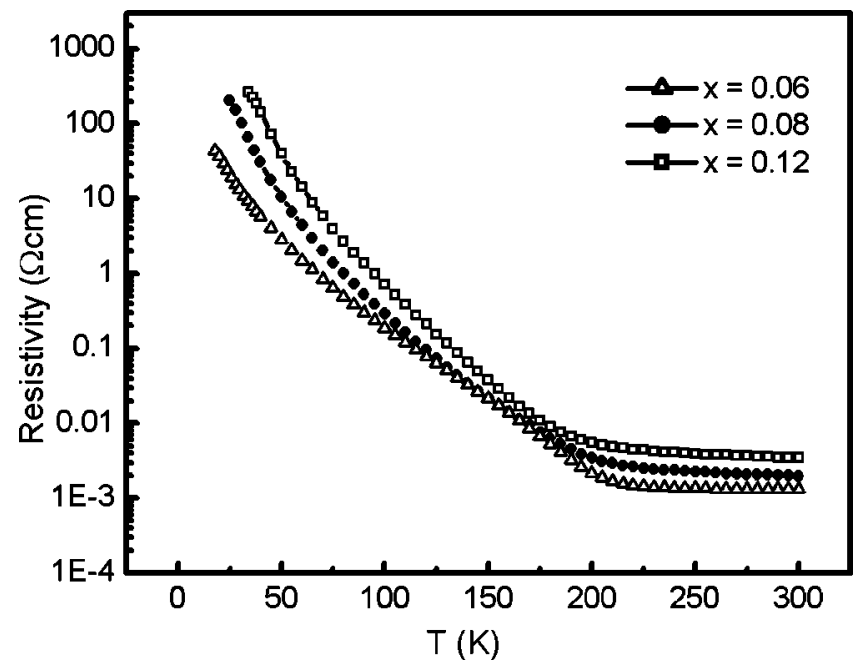

FIG. 4. The temperature dependence of the resistivity for the films, showing semiconducting behavior.

It may appear surprising that Mn-doped Magneli reduced-rutile conducts via holes instead of electrons considering the existence of oxygen vacancies that favor $n$-type conduction. However, in reduced-rutile, the $3 d$ electrons of $\mathrm{Ti}^{3+}$ form a band. The top of the band is probably located near or below the Fermi energy. The current carriers in either situation can be expected to involve holes or a combination of holes and electrons, which is achieved in the former case due to the favorable position of the Fermi level near the top of the band and in the latter case by thermal excitation. Photoemission experiments on $\mathrm{Ti}_{4} \mathrm{O}_{7}$ showed the formation of the $3 d$ band at the Fermi level at room temperature. ${ }^{21}$ The density of states was observed in the band gap on reduced $\mathrm{TiO}_{2}$ surfaces due to $\mathrm{Fe}$ adsorption. ${ }^{30}$

\section{CONCLUSIONS}

In conclusion, room temperature ferromagnetic semiconductor $\mathrm{Mn}_{x} \mathrm{Ti}_{1-x} \mathrm{O}_{2-\delta}(x=0.02-0.12)$ thin films have been successfully grown by pulsed-laser deposition. The magnetic and transport properties of the films have been investigated. The saturation magnetization of Mn-doped reduced film reaches as high as $0.83 \mu_{\mathrm{B}} / \mathrm{Mn}(x=0.08)$ at room temperature. It is decreased to $0.14 \mu_{\mathrm{B}} / \mathrm{Mn}$ at a higher Mn concentration of $x=0.12$. The resistivity shows semiconducting behavior for the doped reduced-rutile films. The carriers are $p$-type. The origin of the holes in the reduced-rutile is discussed. The large hole concentration in our samples may have played an important role in the coupling between the Mn magnetic moments that gives rise to the room temperature ferromagnetism.

\section{ACKNOWLEDGMENTS}

The authors thank Sharp Laboratories of America and Louisiana Board of Regents Support Fund [Grant No. LEQSF (2000-03)-RD-B-10] for supporting this work.
${ }^{1}$ H. Ohno, A. Shen, F. Matsukura, A. Oiwa, A. Endo, S. Katsumoto, and Y. Iyc, Appl. Phys. Lett. 69, 363 (1996).

${ }^{2}$ H. Ohno, J. Vac. Sci. Technol. B 18, 2039 (2000); H. Ohno, F. Matsukura, and Y. Ohno, Jpn. Soc. Appl. Phys. Int. 5, 4 (2002).

${ }^{3}$ M. L. Reed, M. K. Ritums, H. H. Stadelmaier, M. J. Reed, C. A. Parker, S. M. Bedair, and N. A. El-Masry, Mater. Lett. 51, 500 (2001).

${ }^{4}$ M. K. Reed, N. A. El-Master, H. H. Stadelmaier, M. K. Ritums, M. J. Reed, C. A. Parker, J. C. Roberts, and S. M. Bedair, Appl. Phys. Lett. 79, 3473 (2001).

${ }^{5}$ G. T. Thaler et al., Appl. Phys. Lett. 80, 3964 (2002).

${ }^{6}$ S. Sonoda, S. Shimizu, T. Sasaki, Y. Yamamoto, and H. Hori, J. Cryst. Growth 237-239, 1358 (2002).

${ }^{7}$ N. Theodoropoulou, A. F. Hebard, M. E. Overberg, C. R. Abernathy, S. J. Pearton, S. N. G. Chu, and R. G. Wilson, Phys. Rev. Lett. 89, 107203 (2002).

${ }^{8}$ M. E. Overberg et al., J. Vac. Sci. Technol. B 20, 969 (2002).

${ }^{9}$ G. A. Medvedkin, T. Ishibashi, T. Nishi, and K. Hiyata, Jpn. J. Appl. Phys., Part 2 39, L949 (2000).

${ }^{10}$ S. Cho et al., Phys. Rev. Lett. 88, 257203 (2002).

${ }^{11}$ G. A. Medvedkin, K. Hirose, T. Ishibashi, T. Nishi, V. G. Voevodin, and K. Sato, J. Cryst. Growth 236, 609 (2002).

${ }^{12}$ K. Ueda, H. Tabata, and T. Kawai, Appl. Phys. Lett. 79, 988 (2001).

${ }^{13}$ S. A. Chambers, Mater. Today 5, 34 (2002); S. A. Chambers, S. Thevuthasan, R. F. C. Farrow, R. F. Marks, J. U. Thiele, L. Folks, M. G. Samant, A. J. Kellock, N. Ruzycki, D. L. Ederer, and U. Diebold, Appl. Phys. Lett. 79, 3467 (2001).

${ }^{14}$ Y. Matsumoto, M. Murakami, T. Shono, T. Hasegawa, T. Fukumura, M. Kawasaki, P. Ahmet, T. Chikyow, S. Koshihara, and H. Koinuma, Science 291, 854 (2001).

${ }^{15}$ In-Bo Shim, Sung-Yong An, Chul Sung Kim, Se-Young Choi, and Yong Wook Park, J. Appl. Phys. 91, 7914 (2002).

${ }^{16}$ W. K. Park, R. J. Ortega-Hertogs, J. S. Moodera, A. Punnoose, and M. S. Seehra, J. Appl. Phys. 91, 8093 (2002).

${ }^{17}$ Y. Matsumoto, R. Takahashi, M. Murakami, T. Koida, X. J. Fan, T. Hasegawa, T. Fukumura, M. Kawasaki, S. Y. Koshihara, and H. Koinuma, Jpn. J. Appl. Phys., Part 2 40, L1204 (2001).

${ }^{18}$ Z. Wang, W. Wang, J. Tang, L. D. Tung, L. Spinu, and W. Zhou, Appl. Phys. Lett. 83, 518 (2003).

${ }^{19}$ S. Andersson and L. Jahnberg, Ark. Kemi 21, 413 (1964); S. Anderson, B. Collen, V. Kuylenstierna, and A. Magneli, Acta Chem. Scand. 11, 1941 (1957).

${ }^{20}$ J. B. Goodenough, in Progress in Solid State Chemistry, Vol. 5, edited by H. Reiss (Pergamon Press, New York, 1971), p. 145.

${ }^{21}$ M. Abbate, R. Potze, G. A. Sawatzky, C. Schlenker, H. J. Lin, L. H. Tjeng, C. T. Chen, D. Teehan, and T. S. Turner, Phys. Rev. B 51, 10150 (1995).

${ }^{22}$ The ferromagnetism in Mn-Ti is subject to debate. See E. K. Jeong, J. C. Holzer, A. E. Carlsson, M. S. Conradi, P. A. Fedders, and K. F. Kelton, Phys. Rev. B 41, 1695 (1990); and N. Ali, F. Willis, J. C. Holzer, X. Zhang, and K. F. Kelton, J. Appl. Phys. 69, 5136 (1991).

${ }^{23}$ T. Dietl, H. Ohno, F. Matsukura, J. Cibert, and D. Ferrand, Science 287, 1019 (2000).

${ }^{24}$ J. König, H.-H. Lin, and A. H. MacDonald, Phys. Rev. Lett. 84, 5628 (2000).

${ }^{25}$ M. Berciu and R. N. Bhatt, Phys. Rev. Lett. 87, 107203 (2001).

${ }^{26}$ S. J. Potashnik, K. C. Ku, R. Mahendiran, S. H. Chun, R. F. Wang, N. Samarth, and P. Schiffer, Phys. Rev. B 66, 012408 (2002).

${ }^{27}$ H. Ohno, A. Shen, F. Matsukura, A. Oiwa, A. Endo, S. Katsumoto, and Y. Iye, Appl. Phys. Lett. 69, 363 (1996).

${ }^{28}$ S. Oseroff and P. H. Keesom, in Diluted Magnetic Semiconductors, edited by J. K. Furdyna and J. Kossut (Academic Press, New York, 1988).

${ }^{29}$ Y. Shapira, in Semimagnetic Semiconductors and Diluted Magnetic Semiconductors, edited by M. Averous and M. Balkanski (Plenum, New York, 1990).

${ }^{30}$ U. Diebold, H.-S. Tao, N. D. Shinn, and T. E. Madey, Phys. Rev. B 50, 14474 (1994). 